TITLE:

\title{
Water adsorption process of bamboo heated at low temperature
}

$\operatorname{AUTHOR}(S)$ :

Ohmae, Yoshimi; Saito, Yukie; Inoue, Masafumi; Nakano, Takato

CITATION:

Ohmae, Yoshimi ...[et al]. Water adsorption process of bamboo heated at low temperature. Journal of Wood Science 2008, 55(1): 13-17

ISSUE DATE:

2008-11

URL:

http://hdl.handle.net/2433/141955

\section{RIGHT:}

The final publication is available at www.springerlink.com; This is not the published version. Please cite only the published version.; この論文 は出版社版でありません。引用の際には出版社版をご確認ご利用くだ さい。 
Water adsorption process of bamboo heated at low temperature

Yoshimi Ohmae $^{1}$, Yukie Saito ${ }^{1}$, Masafumi Inoue ${ }^{2}$ and Takato Nakano ${ }^{3^{*}}$

1: Graduate School of Agriculture and Life Sciences, The University of Tokyo, Bunkyou-ku

Yayoi 1-1-1, Tokyo, 113-8657.

2: Asian Natural Environmental Science Center, The University of Tokyo, Bunkyou-ku Yayoi

1-1-1, Tokyo, 113-8657.

3: Laboratory of Biomaterials Design, Division of Forest and Biomaterials Science, Graduate

School of Agriculture, Kyoto University, Kita-Shirakawa, Kyoto, 606-8502 Japan.

* Corresponding Author. phone: +81-75-753-9234. fax: +81-75-753-9234.

E-mail address: tnakano@kais.kyoto-u.ac.jp (T. Nakano). 


\section{Abstract}

The water adsorption capacities of moso bamboo samples heated at $200^{\circ} \mathrm{C}$ for various times were evaluated following their conditioning in a closed container at $97 \%$ relative humidity $(\mathrm{RH})$ at $20^{\circ} \mathrm{C}$. Logistic regression analysis was used for curve fitting to the adsorption data and its parameters were analyzed. These parameters were compared with those derived previously from the Dubinin and Radushkevich theory. The properties of the heat-treated samples changed after $5 \mathrm{hr}$ heating.

Before $5 \mathrm{hr}$ heating, hydroxyl groups provided the main adsorption sites but their numbers decreased on heating. After $5 \mathrm{hr}$,-gasification of the bamboo increased and capillaries formed.

Keywords: Water, Adsorption, Adsorption process, Heating, Bamboo, Logistic Regression Analysis, Dubinin - Rudshkevich Theory 


\section{Introduction}

Like wood, bamboo is a component of the forest biomass. However, the industrial potential

of this resource has not been studied in depth. The properties of bamboo can be changed by heat

treatment, and it is well known that both heat-treated wood and bamboo provide useful adsorbents as

used in humidity conditioning. ${ }^{1-6}$ The changes in the ultrastructure of bamboo upon heating are not

well understood, but important changes occur in the water adsorption capacity of bamboo with

increases in the time of heat treatment. The mechanism of these changes in adsorption properties can

be examined using samples subjected to mild heating only. An understanding of the ultrastructural

changes involved may facilitate exploitation of this little-used portion of the forest biomass.

We examined the water adsorption capacity of bamboo samples heated at $200^{\circ} \mathrm{C}$, which is a

relatively low temperature for heat treatment. The results obtained with different heating time were

presented in our previous report where we examined the changes in water adsorption by analyzing

the isothermal responses of different samples ${ }^{6}$. In the present study, the adsorption processes of these

samples were examined. The samples were conditioned at $20^{\circ} \mathrm{C}$ under conditions of $97 \%$ relative

humidity (RH), and their moisture contents were measured over time. Logistic regression analysis 
was applied to the results, and equation parameters were analyzed and compared with those derived

from the Dubinin and Radushkevich theory.

\section{Experimental}

The same samples of 6-year-old moso bamboo (Phyllostachys pubescens; harvested in

October 2004 in Shimane Prefecture in Japan) as used in our earlier study were used here. The

bamboo culm consisted of 27 internodes designated No. 1 to 27 starting from the bottom to the top of

the culm. Block samples were prepared from the center of internodes No. 14 and 15 from the middle

of the culm. Each block had rectangular dimensions of $20(\mathrm{~L}) \times 5(\mathrm{R}) \times 5(\mathrm{~T})(\mathrm{mm})$.

The samples were dried at $105^{\circ} \mathrm{C}$ for $24 \mathrm{hr}$ prior to heat treatment and then heated at $200^{\circ} \mathrm{C}$

for 0 (untreated sample used as a control), $1(\ln 1=0), 2(\ln 2=0.69), 3(\ln 3=1.10), 5(\ln 5=1.61), 20$

$(\ln 20=3.00), 48(\ln 48=3.88), 72(\ln 72=4.28), 120(\ln 120=4.79), 240(\ln 240=5.48)$, or $360 \mathrm{hr}$

$(\ln 360=5.89)$. Heating times were determined to allow the 11 data points to be plotted at nearly

regular intervals on a logarithmic scale. Weight loss due to heat treatment was estimated by

reference to the weight of the unheated weight dried at $100^{\circ} \mathrm{C}$. 
Samples were placed in a desiccator with a saturated solution of $\mathrm{K}_{2} \mathrm{SO}_{4}$ to provide $97 \% \mathrm{RH}$

and their weight were measured at $20^{\circ} \mathrm{C}$ for the duration of the experiment. The weight of each sample was plotted against adsorption time on a logarithmic scale. The adsorption data obtained were then analyzed using logistic regression analysis to extract the descriptive parameters.

\section{Results and Discussion}

\subsection{Regression Curves of Adsorption Process}

The results of adsorption by the heat-treated bamboo are shown in Figure 1. Regression curves obtained through fitting a logistic equation to the data are shown as solid lines. All the regression curves showed excellent agreement with the observed absorption data values lending support to the efficacy of the logistic equation to model the adsorption process of the bamboo samples. The values given by the regression curve almost agreed with experimental data for each heat-treated sample, stabilized sample obtained under the same isothermal experimental conditions.

The logistic equation used is the solution of the nonlinear differential equation was as

follows: 


$$
\frac{d y}{d x}=\frac{a}{b} y(b-y) \quad(\mathrm{y}=m c, x=\ln (t))
$$

This equation may be transformed into:

$$
\frac{d y}{d x}=-\frac{a}{b}\left(y-\frac{b}{2}\right)^{2}+\frac{a b}{4}
$$

The maximum value of adsorption rate $d y / d x$ was then given by ab/4 and the solution of equation (1)

was:

$$
y=\frac{b}{1+c \exp [-a x]}
$$

As equation (3) can be transformed into $1 / y=(1 / b)+(c / b) \exp [-a x]$, parameters $a, b$, and $c$ may be determined easily. These parameters characterize the curves in the absorption process described by equation (3).For an equilibrium moisture content, $b$ denotes the value when the curve is stabilized, i.e., the equilibrium moisture content, $a b / 4$ is the maximum adsorption rate, and $\ln [c] / a$ is the time required to achieve this maximum adsorption rate. Hence, water adsorption in bamboo can be described by $b$ the leveling off value $b$ when the regression curve is stabilized, $a b$ the maximum adsorption rate, and $\ln [c] / a$ the time taken to achieve this maximum adsorption rate. Variations in these parameters enable characterization of the differences in the adsorption process resulting from different heating times. 
It should be noted that these parameters do not correspond directly to physical properties of each heated sample. Rather, the parameters describe the character of the adsorption process for each sample. As the parameters do not relate directly to structural changes in the bamboo or other physical factors resulting from heat treatment, other considerations are needed to account for the behavior of parameters in the adsorption process.

The logistic curves derived from the observed values of each heat-treated sample and the corresponding differential curves are shown in Figure 2. The differential curves are normalized to make the maximum rate of adsorption equal to unity. The logistic curves shown in Figure 2 suggest that they may be placed into two groups: those heated for less than 3-5 hr and for more than this time.

The differential curves lend support to this notion and show that the peak corresponding to the maximum rate of adsorption shifts to shorter time for samples heated for more than 3-5 hr.

\subsection{Dependence of Parameters on Heating Time}


Figure 3 shows the relationships between parameters $a, b$, and $c$ and weight loss. An inflexion point occurred upon heating for 3-5 hr. This point corresponds with that found from analysis of isotherms, as reported in our previous paper ${ }^{6}$.

The behavior of the equilibrium moisture content, denoted here by $b$, was reported elsewhere $^{6}$. In this previous study, we showed that the equilibrium moisture content against heating time causes the minimum peak after heating for $2-5 \mathrm{hr}$ as a result of changes in both the chemical and physical structure. Two parameters are used to describe the adsorption curves, $a b$ and $\ln [t]$.

Figure 4 shows $a b$ against weight loss upon heating. Parameter $a b$ decreases on heating for up to $5 \mathrm{hr}$ and then increases following further heating. In contrast, Figure 5 shows that the time required to reach maximum adsorption rate during the adsorption experiments, $\ln [t]$, decreased after a peak at $3 \mathrm{hr}$. These two parameters appear to contribute to weight loss in opposite ways. The relationship between them is shown in Figure 6, which shows how heating time affects the adsorption process. The maximum adsorption rate parameter, $a b$, decreased markedly prior to 3-5 hr, but the corresponding parameter $\ln [t]$ showed little change. Over longer heating times, the parameter $a b$ decreased linearly with $\ln [t]$. 
Considering changes in adsorption behavior with heat treatment reported previously ${ }^{6}$, the

results shown in Figures 5 and 6 indicate qualitatively that changes in both the chemical composition and ultrastructure of bamboo can affect adsorption behavior. The two factors responsible can be summarized as a decrease in the hydroxyl groups that provide adsorption sites and the formation of capillaries due to gasification of the bamboo. The results shown in Figure 6 suggest that the former plays the main role in samples heated for less than $5 \mathrm{hr}$, with the latter affecting samples heated for over $5 \mathrm{hr}$.

\subsection{Logistic equation parameters compared with those derived from the Dubinin and}

\section{Radushkevich theory}

In our previous study ${ }^{6}$, the isotherms resulting from bamboo heated to $200^{\circ} \mathrm{C}$ in air were

analyzed using the Hailwood and Horrobin theory and Dubinin and Radushkevich theory ${ }^{7-9}$, and the parameters describing the adsorption characteristics were evaluated. The parameters derived in the present study show an inflection point at a heating time of about $5 \mathrm{hr}$ and hence are correlated with those reported previously. Therefore, they may be compared. The Dubinin and Radushkevich 
parameter $m_{0}$ is related to the amount of saturated adsorption, i.e., the micropore volume, and $E_{0}$ is a

characteristic of the interaction energy.

Figure 7 shows a plot of $a b$ and $\ln [t]$ against $m_{0}$. This figure shows that parameter $a b$

increases and parameter $\ln [t]$ decreases with $m_{0}$ for sample heated for more than $5 \mathrm{hr}$. Hence, as may

be expected, an adsorbate with large micropore volume, and hence large saturated adsorption

capacity, will have a large rate of adsorption. Both parameters show different trends at $5 \mathrm{hr}$, which

can be attributed to a change in the properties of heat-treated moso bamboo after $5 \mathrm{hr}$, as discussed

previously. At this point, the numbers of hydroxyl groups, and hence potential adsorption sites, will

have decreased while gasification of the bamboo began to increase with formation of micropores ${ }^{10}$.

These changes in bamboo as an adsorbent due to heating time divide the plot into two parts. $\ln [t]$ also

corresponds to the heating time of maximum rate of adsorption.

Plots of $a b$ and $\ln [t]$ against parameter $E_{0}$ derived from Dubinin and Radushkevich theory

are shown in Figure 8, and indicate that $a b$ decreases with increasing $E_{0}$ with the trend of the plot

changing again at $5 \mathrm{hr}$. The plots of $\ln [t]$ vs. $E_{0}$ and $m_{0}$ show opposite trends but both show inflection

at $5 \mathrm{hr}$. 
Comparison between the parameters of the logistic equation and those of Dubinin and

Radushkevich theory showed that the rate of water adsorption is strongly dependent on the

parameters $E_{0}$ and $m_{0}$. In our previous report, we noted that $E_{0}$ decreases and $m_{0}$ increases upon

further heating. Presumably, the adsorption rate increases with further changes in ultrastructure

under more severe conditions. 


\section{References}

1. Abe I, Hitomi M, Ikuta N, Kera Y (1996) Pore structural analysis of charcoals by mercury intrusion porosimetry. Tanso 172: 77-82

2. Abe I, Kawafune I, Kera Y (1996) Properties of porous carbons prepared from the wood of Japanese cypress - effect of carbonization time at $900^{\circ} \mathrm{C}$. Tanso 171: 18-23

3. Abe I, Fukuhara T, Maruyama J, Tatsumoto H., Iwasaki S (2001) Preparation of carbonaceous adsorbents for removal of chloroform from drinking water Carbon 39: 1069-1073

4. Abe I., Fukuhara T, Maruyama J, Iwasaki S, Yasuda K, Nakagawa K, Iwata Y, Kominami H, Kera Y (2001) Development of a high density carbonaceous adsorbent from compressed wood.

Carbon 39: 1485-1490

5. Fujiwara S, Sima K, Ciba K (2003) Carbonization condition and gas adsorption characteristics of the bamboo charcoal. Bamboo J 20: 68-76

6. Ohmae Y, Saito Y, Inoue M, Nakano T (2007) Water adsorption capacity of heated bamboo at lower temperature. (in press)

7. Dubinin MM, Radushkevich LV (1947) Dokl. Akad. Nauk SSSR, 55,331. 
8. Dubinin MM, (1960) The potential theory of adsorption of gases and vapors for adsorptions with energetically nonuniform surfaces. Chem Rev. 60: 235-241

9. Dubinin MM (1965) Theory of the bulk saturation of microporous activated charcoals during adsorption of gases and vapors. Russian J Phys Chem. 39: 697-704

10. Nakano T (2003) Surface fractal dimensionality and hygroscopicity for heated wood.

Holzforschung 57: 289-294 


\section{Captions}

Fig. 1. Experimental data and relevant regression curves for samples heated at various temperatures.

Fig. 2. Regression curves and their normalized differential curves for samples heated for 0 to $408 \mathrm{hr}$.

Fig. 3. Weight loss dependence of the parameters $a, b$, and $c$ of regression curves derived from the

logistic function $f(x)=b /(c+\exp [-a x]): f(x)=m c$ and $x=\ln [t]$.

Fig. 4. Relationship between the parameter $a b$ describing the maximum adsorption rate and weight

loss.

Fig. 5. Relationship between the time to achieve maximum adsorption rate, $\ln [t]$, and weight loss.

Fig. 6. Relationship between the parameter $a b$ describing the maximum adsorption rate and the time to achieve the maximum adsorption rate, $\ln [t]$. 
Fig. 7 Relationship between the parameters $a b$ and $\ln [t]$ at $m c=b / 2$ and the parameter $m_{0}$ derived

from the Dubinin and Radushkevich theory. $m_{0}$ describes the limiting volume of the adsorption

space i.e., the saturated moisture content of the capillaries.

Fig. 8. Relationship between parameters $a b$ and $\ln [t]$ at $\mathrm{mc}=\mathrm{b} / 2$ and the parameter $E_{0}$ derived from

Dubinin and Radushkevich theory. $E_{0}$ is related to the characteristic adsorption energy. 


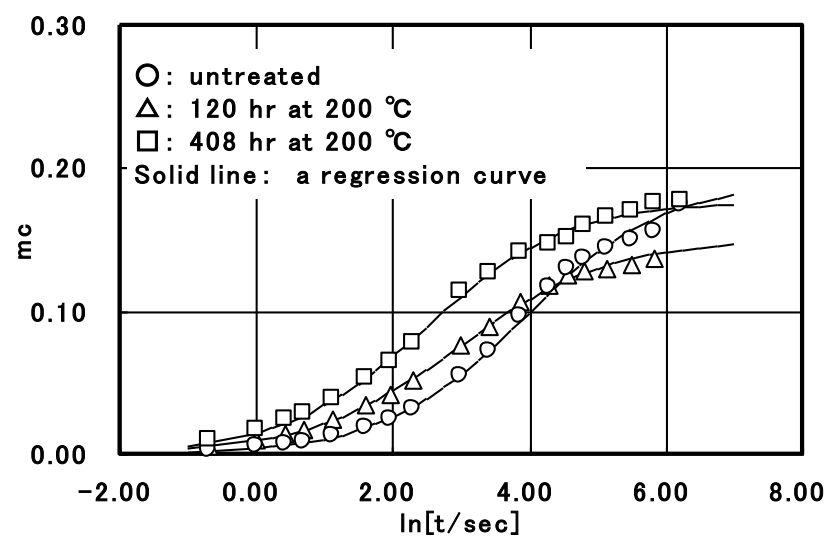

Fig. 1. Experimental data and relevant regression curves for samples heated at various temperatures. 

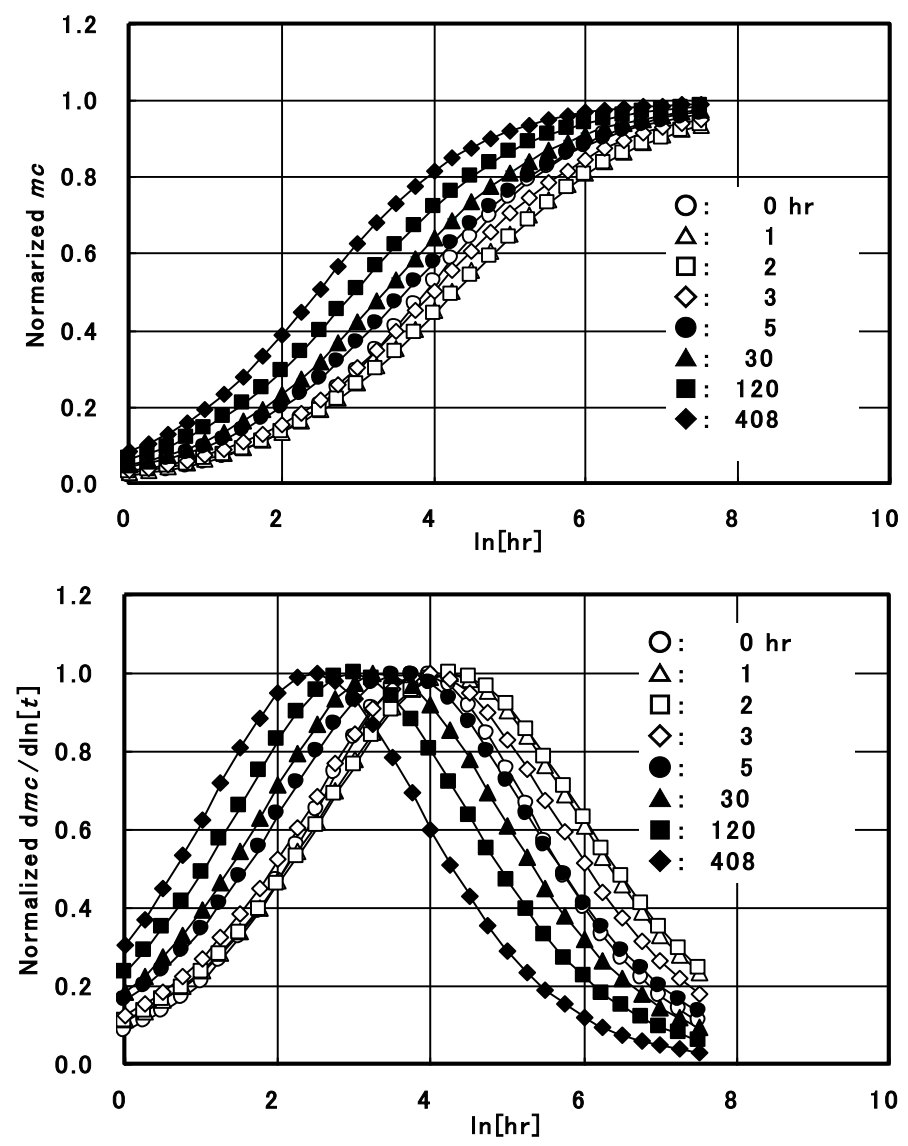

Fig. 2. Regression curves and their normalized differential curves for samples heated for 0 to $408 \mathrm{hr}$. 

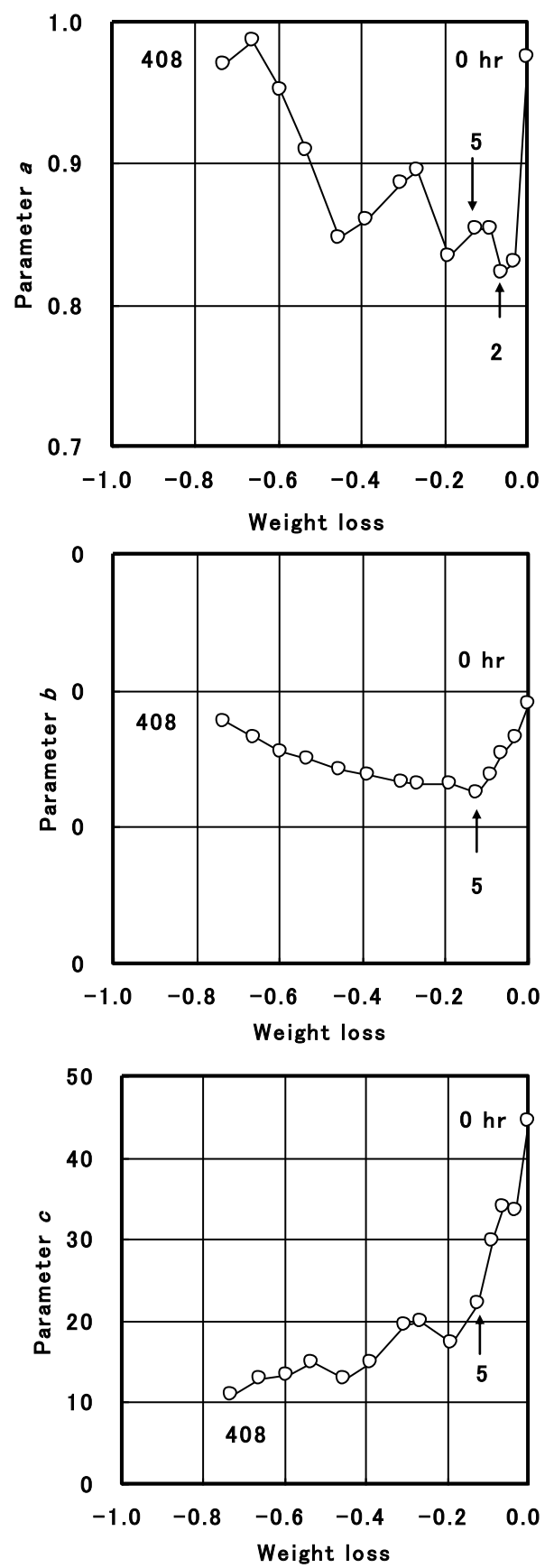

Fig. 3. Weight loss dependence of the parameters $a, b$, and $c$ of regression curves derived from the logistic function $f(x)=b /(c+\exp [-a x]): f(x)=m c$ and $x=\ln [t]$. 


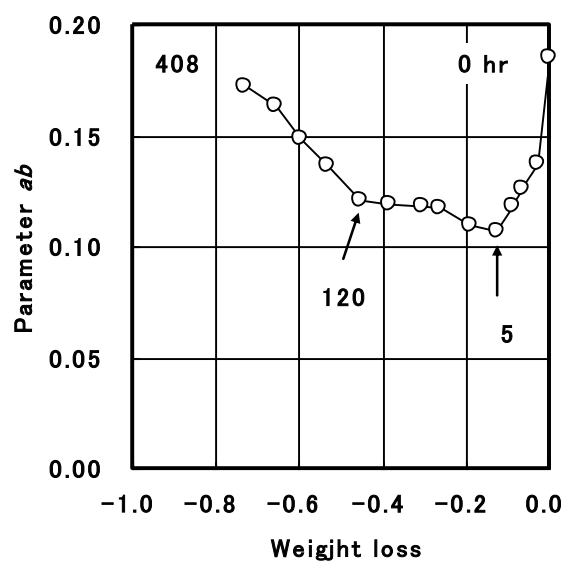

Fig. 4. Relationship between the parameter $a b$ describing the maximum adsorption rate and weight loss. 


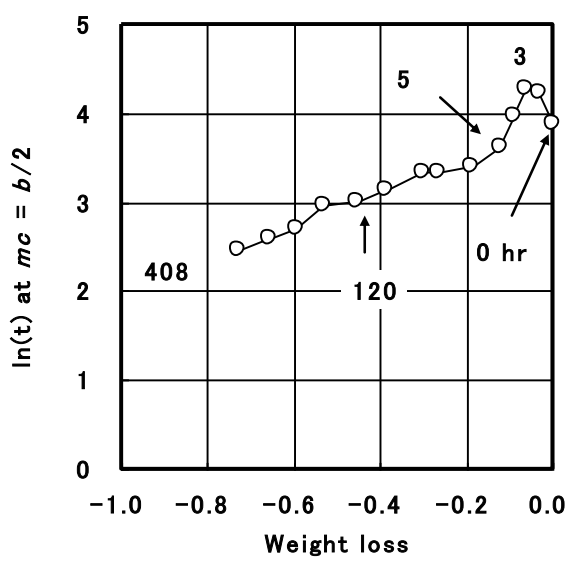

Fig. 5. Relationship between the time to achieve maximum adsorption rate, $\ln [t]$, and weight loss. 


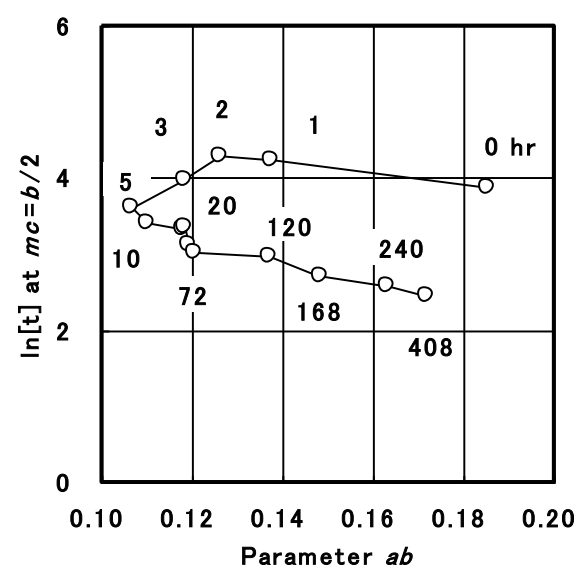

Fig. 6. Relationship between the parameter $a b$ describing the maximum adsorption rate and the time to achieve the maximum adsorption rate $\ln [t]$. 

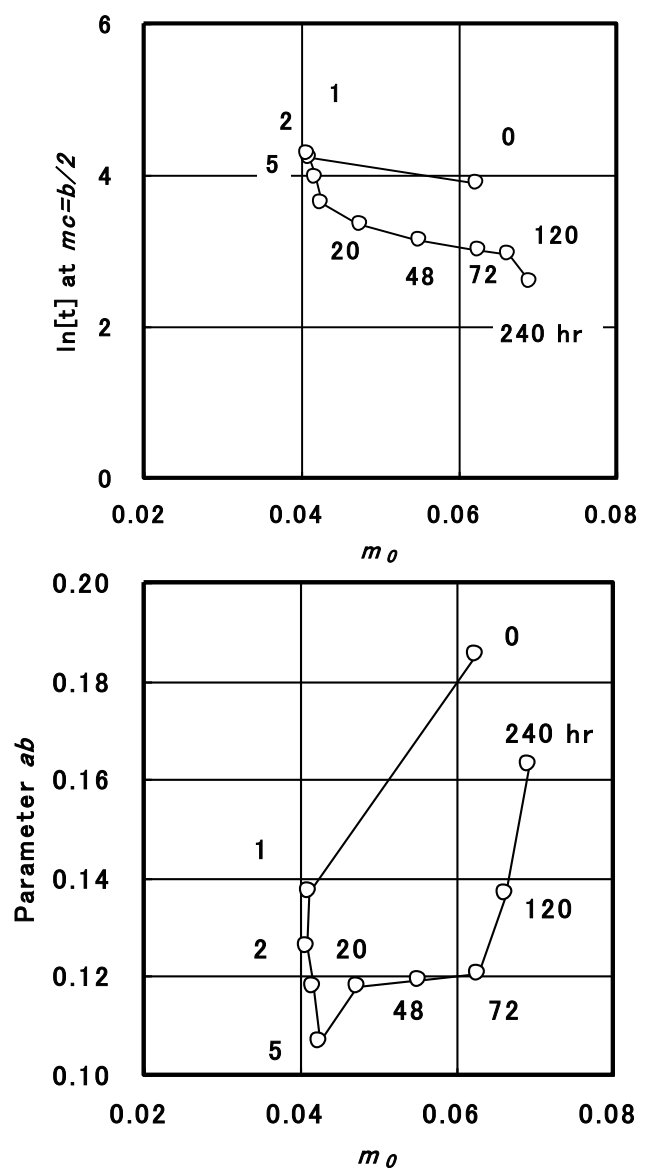

Fig. 7 Relationship between the parameters $a b$ and $\ln [t]$ at $m c=b / 2$ and the parameter $m_{0}$ derived from the Dubinin and Radushkevich theory. $m_{0}$ describes the limiting volume of the adsorption space i.e., the saturated moisture content of the capillaries. 

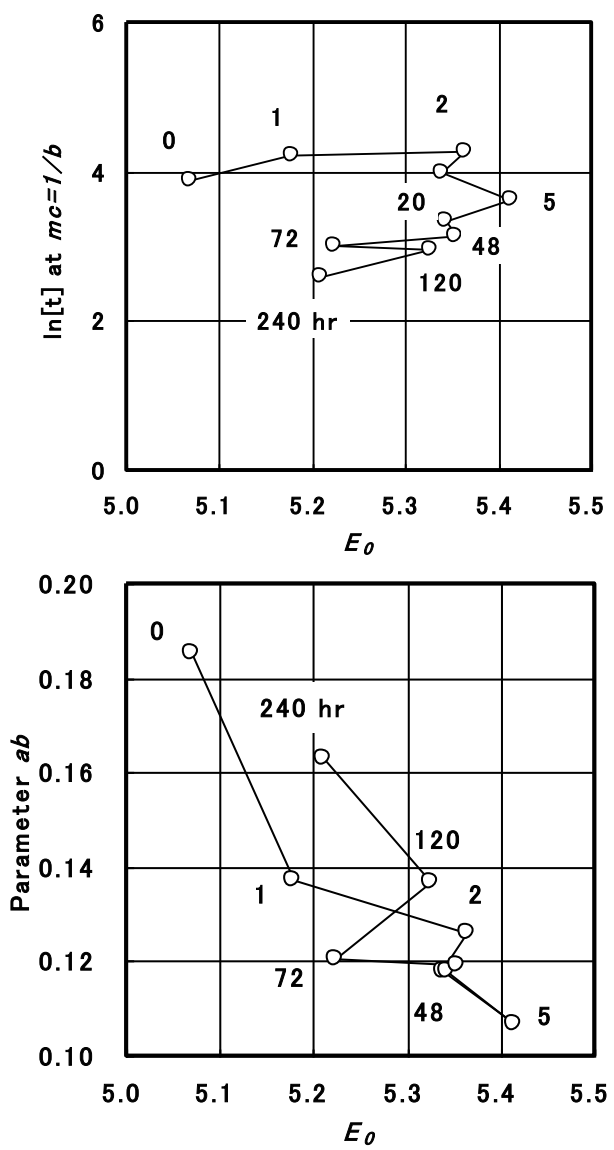

Fig. 8. Relationship between parameters $a b$ and $\ln [t]$ at $m c=b / 2$ and the parameter $E_{0}$ derived from

Dubinin and Radushkevich theory. $E_{0}$ is related to the characteristic adsorption energy. 